\title{
Etiological Analysis of Late Onset Epilepsy at R.I.M.S, Ranchi, Jharkhand, India
}

\author{
Dr. Jag Mohan Kumar ${ }^{1}$, Dr. Umesh Prasad ${ }^{2}$, Dr. Kumari Madhu ${ }^{3}$ \\ ${ }^{I}$ Department Of Medicine/Junior Resident/Rajendra Institute Of Medical Sciences/Ranchi University/India \\ ${ }^{2}$ Department Of Medicine/Associate Professor/Rajendra Institute Of Medical Sciences/Ranchi University/India \\ ${ }^{3}$ Department Of Surgery/Junior Resident/Rajendra Institute Of Medical Sciences/Ranchi University/India
}

\begin{abstract}
Introduction:Epilepsy describes a condition in which a person has recurrent seizures due to chronic underlying process. Epilepsy refers to a clinical phenomenon than a single disease entity, since there are many forms and causes of epilepsy.

Objective:Toanalyse the etiological agents of late onset epilepsy.

Method:Data for the study was collected from patients with epilepsy admitted in Department of Medicine at R.I.M.S RANCHI. Total of 60 patients were included in this study and their detailed etiological analysis was done.

Conclusion:Neurocysticercosis was the most common cause of late onset epilepsy at R.I.M.S, RANCHI in this study.

Keywords:Epilepsy,LateOnset,Neurocysticercosis.
\end{abstract}

\section{Introduction}

Epilepsy describes a condition in which a person has recurrent seizures due to chronic underlying process. Epilepsy refers to a clinical phenomenon than a single disease entity, since there are many forms and causes of epilepsy.(1) brain.(1)

A seizure is a paroxysmal event due to abnormal excessive or synchronous neuronal activity in the seize".

The term epilepsy comes from the greek word " epilambanein" which means "to take hold of " or "to

The worldwide prevalence rate of epilepsy has been estimated at 5-10 persons per 1000.(1),(6).

The prevalence rate in india is 5.59 per 1000 with no stastically different rates between men and women or urban and rural residence.(2),(5).

The incidence of epilepsy is $0.3-0.5 \%$ in different populations throughout the world.(1),(6).

Incidence rate varies from 38 to 49.3 per 1,00,000 population per year from two community based studies in india.(3),(5).

\section{Classification of seizures (1),(7),(8)}

(The International league against epilepsy , commission on classification and terminology)

1. Focal seizures

(can be further described as having motor, sensory, autonomic, cognitive or other features)

2. Genaralised seizures

a. Absence

Typical

Atypical

b. Tonic clonic

c. Clonic

d. Tonic

e. Atonic

f. Myoclonic

3. May be focal, generalized or unclear

Epileptic spasms

Epilepsy of late onset may be simply defined as epilepsy beginning in adult life.Epilepsy beginning in adult life is likely to be due to progressive brain disease as compared to idiopathic epilepsy, which has its onset in childhood or youth.(4),(9),(10). 
Hence this study is aimed to evaluate the etiological analysis of lateonset epilepsy in patients of more than 18 years of age at R.I.M.S, Ranchi.

\section{Materials And Methods}

\section{Source of data:}

Patients admitted in R.I.M.S, Ranchi in the Department of Medicine, in the study period between October 2013 to October 2014.

\section{Inclusion criteria:}

1. Age of onset of epilepsy $>18$ YEARS.

2. Epilepsy diagnosed according to International League Against Epilepsy.

\section{Exclusion criteria:}

1. Onset before 18 years of age but continued to have seizures even after 18 years.

2. Age of onset of seizures $<18$ years.

3. Metabolic seizures

4. Posttraumatic seizures

5. pseudoseizures

Detailed history was taken from the selected patients, clinical examination was done, and following investigations were done:CBC, RBS, Blood urea, serum creatinine,serumsodium, potassium,calcium,magnesium,HIV 1\&2, VDRL,X-Ray,CT Scan/MRI Brain, CSF Analysis, ELISA(Serum/CSF), Quantiferon TB Gold Assay, EEG

\section{Results}

Total 60 patients were taken in this study and following result was found:

\section{Etiology Of Epilepsy}

\begin{tabular}{|l|l|l|}
\hline Etiology & No. Of Patients & Percentage \\
\hline Neurocysticercosis & 30 & $50 \%$ \\
\hline Tuberculoma & 12 & $20 \%$ \\
\hline Idiopathic & 8 & $13.33 \%$ \\
\hline Post Stroke Epilepsy & 6 & $10 \%$ \\
\hline Brain Tumour & 2 & $3.33 \%$ \\
\hline Brain Abscess & 2 & $3.33 \%$ \\
\hline
\end{tabular}

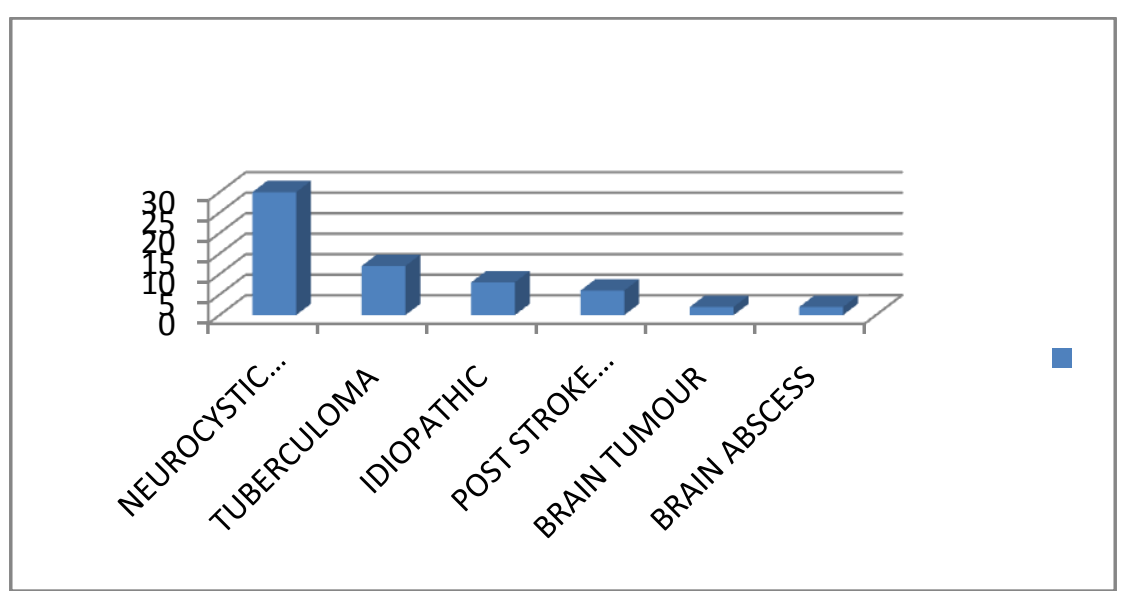

Etiology of generalised seizure:

\begin{tabular}{|l|l|}
\hline Idiopathic & 8 \\
\hline Neurocysticercosis & 6 \\
\hline Post Stroke Epilepsy & 5 \\
\hline Tuberculoma & 3 \\
\hline Brain Abscess & 2 \\
\hline Brain Tumour & 1 \\
\hline
\end{tabular}




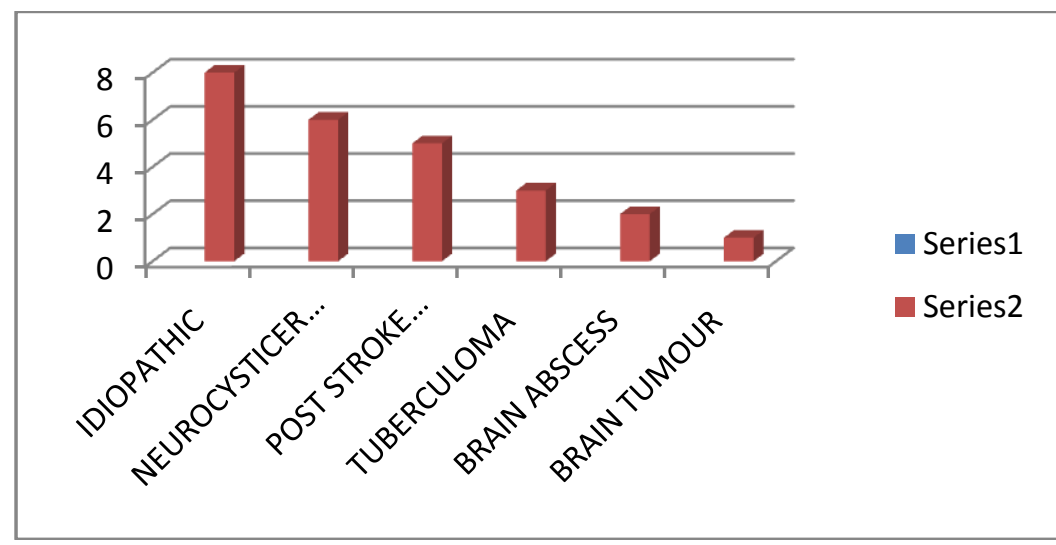

Etiology of partial seizure:

\begin{tabular}{|l|l|}
\hline Neurocysticercosis & 24 \\
\hline Tuberculoma & 9 \\
\hline Post Stroke Epilepsy & 1 \\
\hline Brain Abscess & 1 \\
\hline
\end{tabular}

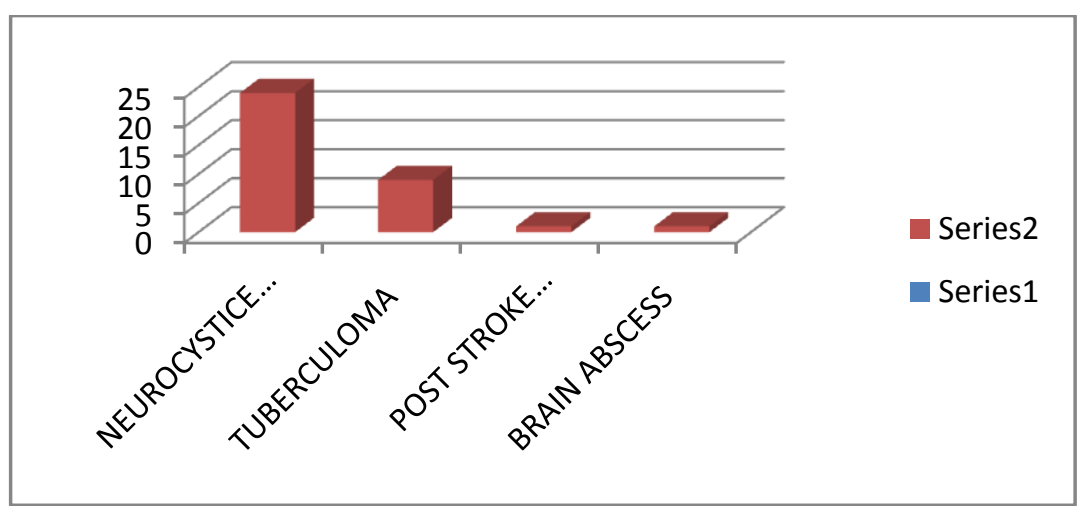

IV. Discussion And Conclusion

Epilepsy beginning in adult life are likely to be an identifiable cause as compared to those beginning in childhood which are more likely to be idiopathic.

In this study, neurocysticercosis $(50 \%)$ was the commonest cause, followed by tuberculoma (20\%), idiopathic (13.33\%), post stroke epilepsy (10\%), brain tumour (3.33\%), brain abscess (3.33\%).

In the study performed by Perez Lopez JL, Longo $J$, et al. Late onset epileptic seizures.ActaNeurologicaScandinavica 1985;72(4):380-4 among 50 patients, 16 patients (32\%) etiology could not be ascertained.Among the 34 symptomatic patients $(68 \%), 16$ patients $(47.05 \%)$ had post stroke epilepsy, 1 patient (2.94\%) had neurocysticercosis, 3 patients $(8.84 \%)$ had tumour, 3 patients $(8.84 \%)$ had metastasis, 7 patients (20.58\%) had metabolic etiology and 4 patients (11.76\%) had infective etiology.(9)

As per the study of Pradeep PV, Balasubramanian R, RaoSN.Clinical profile and etiological analysis of late onset epilepsy. JAPI 2003;51:1192 etiology of seizure was Idiopathic(44\%), Cerebrovascular accident (20\%), Neurocysticercosis (12\%), Tumour (4\%), Vascular malformation (4\%), Mesial temporal lobe epilepsy (4\%).(4).

As per the study of Srinivas P, Prasad Rajendra R, NaikVasudevaH,SreenivasaM, Suresh K. New onset seizures in adults: etiological and clinical profile. JAPI 2003;51:1191, Etiology of epilepsy was cerebrovascular accident $(40 \%)$, space occupying lesion(12\%), metabolic(12\%).(11).

This variation in study might be due to geographical variation, low socioeconomic status and low rate of literacy prevalent in this region.

Further studies in this region are required to corroborate with the findings of this study.

\section{References}

[1]. Daniel HL, Seizures and Epilepsy. In :Dan Longo,DennisL.Kasper, J. Larry Janeson, Antony S. Fauci, Stephen L. Hauser, Joseph Loscalzo, Harrison's Principles of internal Medicine. 18 ${ }^{\text {th }}$ ed. McGraw Hill,2012.P:3251-3270

[2]. Sridaharan R, Murthy BN. Prevalence and Pattern of Epiliepsy in India. Epilepsia 1999;40(5):631-6.

[3]. Radha Krishnan K, Pandian JD. Prevalence, Knowledge attitude and practice of epilepsy in kerala, south India. Epilepsia 2000;41(8):1027-35 
[4]. Pradeep PV, BalasubramaniamP,Rao SN, Clincal profile and etiological analysis of late onset epilepsy. JAPI 2003;51:1192.

[5]. Ray BK, Bhattacharya S. Epidemiology OF Epilepsy-Indian perspective. J Indian Med Assoc 2002;100(5):322-326

[6]. Sander JW. The epidemiology of epilepsy revisited. Current opinion in Neurology 2003;16(2):165-70

[7]. Commission on classification and terminology of the international league against epilepsy-proposal for revised and electroencephalographic classification of epileptic seizures. Epilepsia 1981;22:489-501.

[8]. Epilepsy and other seizure disorders. In :adams and victor's principles of neurology. $9^{\text {th }}$ edn. Mcgraw hill 2005.p.304-313.

[9]. Perez lopez JL, et al . late onset epileptic seizures. A retrospective study of 150 patients. Acta neurologic scandinovica 1985;72(4):380-384.

[10]. Martinez - Garcia FA, et al. late onset epileptic crisis and cerebrovascular disease. Revista de neurologica 1998;27(158):6.

[11]. Srinivas P, Prasad Rajendra R, NaikVasudeva H, Sreenivasa M, Suresh K. New onset seizures in adults:etiological and clinical profile.JAPI 2003;51:1191. 